\title{
Radiological Assessment in Necrotizing Fasciitis
}

Mustafa C. GÖK ${ }^{1}$, Yalçın TURHAN², Murat DEMIROĞLU³, Bülent KILIÇ4, Mesut AKKUŞ3, Korhan ÖZKAN³

\footnotetext{
${ }^{1}$ Department of Radiology, Baltalimanı Education and Research Hospital, İstanbul, Turkey.

2 Department of Orthopaedics and Traumatology, Medical Faculty, Düzce University, Düzce, Turkey.

${ }^{3}$ Department of Orthopaedics and Traumatology, Medical Faculty, Medeniyet University, İstanbul, Turkey.

${ }^{4}$ Department of Healthcare Management, Faculty of Health Sciences, İstanbul Gelişim University, İstanbul, Turkey.
}

\section{ABSTRACT}

Necrotizing fasciitis is a rarely seen condition that affects the superficial fascia and subcutaneous fatty tissue and can be life threatening. It is mostly encountered in an acute fashion, but subacute progressive course can be seen rarely. Necrotizing fasciitis can affect any part of the body, but it is mostly seen in extremities, especially in the lower extremity. Although some traumatic events such as open fractures, crush injuries, lacerations, steroid injections, insect bites, burns, or frostbite injuries can cause necrotizing fasciitis, it can also be seen without any trauma. Affected extremity or the life of the patient can be protected with rapid diagnosis and treatment. Radiological tests are important as clinical doubt and examination. This study aimed to discuss the importance of the radiological assessment for the diagnosis of this lifethreatening condition in the context of current literature.

Key words: MRI, necrotizing fasciitis, radiology

\section{REVIEW}

Necrotizing fasciitis is a rapidly progressive bacterial infection of the soft tissues and fascia. It can be fatal if the diagnosis and treatment are delayed $(1,2)$. Besides clinical signs, radiological tests play an important role in diagnosis. Radiological tests guide the clinician to detect the complications and disease progression. With the help of these tests, the differentiation of other soft-tissue pathologies that can be misdiagnosed as necrotizing fasciitis can be done easily.

The radiological tests used in the diagnosis include conventional radiography (CR), ultrasonography (US), computerized tomography (CT), and magnetic resonance imaging (MRI). Soft-tissue swellings, deletions in fatty planes, and skin ulcers can be seen in $C R$, but the most important one is the presence of air in the soft tissues (3). The presence of air or foreign bodies suggests infectious processes. The US can show air in the soft tissues or subcutaneous area. Also, deep vein thrombosis and soft-tissue tumors can be identified using US.

Sectional imaging takes a more important place in the diagnosis of necrotizing fasciitis. CT is the most sensitive modality for soft-tissue gas detection. Compared with radiography, $C T$ is superior to evaluate the extent of tissue or osseous involvement. It shows an underlying (and potentially more remote) infectious source and reveals serious complications such as vascular rupture complicating tissue necrosis $(4,5)$. Similarly, the rapidity of CT compared with MRI may be advantageous for evaluating an emergent necrotizing fasciitis.

MRl is the most useful imaging modality in diagnosing necrotizing fasciitis and is more advantageous than (T (6). However, it is not suitable in the emergent cases because necrotizing fasciitis is a rapidly progressive disease and MRI is a timeconsuming procedure (7). 
MRI sequences useful for diagnosing necrotizing fasciitis include T1-weighted fast spin-echo (SE) and T2-weighted fatsuppressed fast SE (6). T1-weighted fat-suppressed gadoliniumdiethylenetriaminepentaacetic acid (Gd-DTPA) contrast-enhanced fast SE sequences can be used when the patients' renal functions are intact. Disruption of muscle tissue patterns and high signal intensities in the muscles because of hemorrhage can be seen in T1-weighted images. Hypointense signals can be seen in T1-weighted images throughout the deep fascia (Figure 1).

Diffuse edema characterized by a high signal in the muscle and subcutaneous fatty tissue, and thickening in the deep fascia and interfascial planes can be seen in T2-weighted images (Figure 2). The thickening in the deep fascia more than $3 \mathrm{~mm}$ is helpful for diagnosis (8). Areas without any signal on T1- and T2-weighted images can be seen in the presence of air in soft tissues and fascia. Gradient echo sequences are more important than others in detecting air presence (6). Some contrast-retaining and unretaining areas can be seen in different stages in contrastenhanced T1-weighted images in affected fascia (9).

Thickening of the fascia more than $3 \mathrm{~mm}$ and involvement of the deep intermuscular fascia and three or more compartments are the signs of fascial involvement in necrotizing fasciitis (3).

In addition to diagnosing necrotizing fasciitis, MRI is also helpful in detecting the disease progression and related complications.

Some diseases can be misdiagnosed as necrotizing fasciitis in the MRI. Non-necrotizing fasciitis (paraneoplastic fasciitis, eosinophilic fasciitis), cellulite, infectious myositis, and pyomyositis can create images similar to necrotizing fasciitis (2). Also, compartment syndrome usually occurring after traumatic injuries can mimic necrotizing fasciitis clinically.

Cellulite is a frequent disease, and the clinical findings can mimic necrotizing fasciitis. It usually involves superficial fascia and dermis; subcutaneous fatty edema and thickening is more evident in the MRI compared with necrotizing fasciitis. The deep intermuscular fascia is highly protected in cellulite, and the presence of gas in the fascia is in favor of necrotizing fasciitis (2).

Radiographs are usually unremarkable, excluding the sequelae of any sustained trauma in compartment syndrome. CT images may reveal intramuscular hypoattenuation from edema. In more

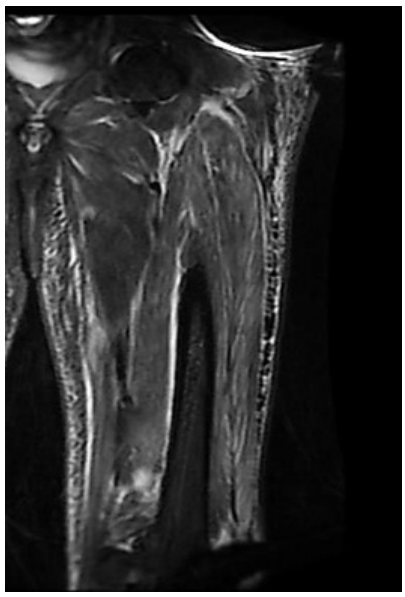

FIGURE 1: A 50-year-old patient with necrotizing fasciitis having air in the subcutaneous fatty tissues in coronal T1-weighted images.

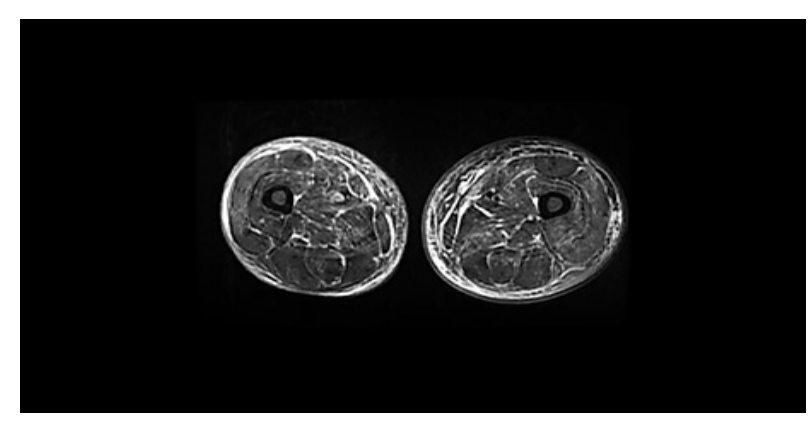

FIgURE 2: A High signals in the superficial and deep fascia in fat-suppressed T2-weighted axial images in the left thigh of a 50-year-old patient with necrotizing fasciitis. Also, subcutaneous edema and air in the soft tissues can be seen.

acute and subacute stages, MRI shows decreased T1 signal intensity and increased $\mathrm{T} 2$ signal intensity within the edematous muscle, loss of normal muscle striations, enlargement of the affected muscle group, and variable signal intensity of any compartmental hemorrhage (depending on the age). Diffuse heterogeneous hypointense $T 1$ and $T 2$ signal intensity is seen in chronic compartment syndrome and muscle necrosis (10).

In conclusion, necrotizing fasciitis is an emergent infectious disease and can cause severe morbidities and mortality in the patients. Early diagnosis and treatment are important. Loss of the affected extremity or the life of the patient can be inevitable in delayed cases. Accordingly, the imaging modalities and their appropriate evaluation of this emergent disease should be known to the clinician because rapid diagnosis and management are crucial. 


\section{REFERENCES}

1. Vayvada H, Demirdöver C, Menderes A, Karaca C. Necrotising fasciitis: diagnosis, treatment and review of the literature. Turkish Journal of Trauma and Emergency Surgery 2012;18(6):507-513.

2. Chauhry AA, Baker KS, Gould ES, Gupta R. Necrotising fasciitis and its mimics: What radiologists need to know. AJR 2015;204(1):128-139.

3. Hayeri MR, Ziai P, Shehata ML, Teytelboym OM, Huang BK. Soft-tissue infections and their imaging mimics: From cellulitis to necrotizing fasciitis. RadioGraphics 2016; 36(6):1888-1910.

4. Struk DW, Munk PL, Lee MJ, Ho SG, Worsley DF. Imaging of soft tissue infections. Radiol Clin North Am 2001;39(2):277303.

5. Wysoki MG, Santora TA, Shah RM, Friedman AC. Necrotizing fasciitis: CT characteristics. Radiology 1997;203(3):859-863.
6. Ali SZ, Srinivasan S, Peh WCG. MRI in necrotising fasciitis of the extremities. Br J Radiol 2013;87(1033):20130560.

7. Cardinal E, Bureau N, Aubin B, Chem R. Role of ultrasound in musculoskeletal infections. Radiol Clin North Am 2001;39(2):191-199.

8. Kim KT, Km JY, Won Lee JW, Park SW, LimMK, et al. Can necrotizing infectious fasciitis be differentiated from nonnecrosing infectious fasciitis with MR imaging? Radiaology 2011;259(3):816-824.

9. Malqhem J, Lecouvet FE, Omoumi $P$, Maldaque BE, Vande Berq BC. Necrotizing fasciitis: contribution and limitations of diagnostic imaging. Joint Bone Spine 2013;80(2):146-154.

10. Konstantakos EK, Dalstrom DJ, Nelles ME, et al. Diagnosis and management of extremity compartment syndromes: an orthopaedic perspective. The American Surgeon 2007;73(12):1199-1209. 